\title{
Augmentation in Restless Legs Syndrome: Treatment with Gradual Medication Modification
}

\author{
Adam Rosenstein, Marcie Rabin and Roger Kurlan*
}

The Atlantic Neuroscience Institute, Overlook Medical Center, 99 Beauvoir Ave. Summit, N.J. 07901

\begin{abstract}
Dopaminergic drugs can cause augmentation during the treatment of restless legs syndrome (RLS). We previously reported that sudden withdrawal of dopaminergic treatment was poorly tolerated. We now report our experience with gradual withdrawal of the dopaminergic drug during the drug substitution process using a retrospective chart review with comparison to previous data. Seven patients with RLS and dopaminergic drug-induced augmentation were treated with a gradual withdrawal of the offending drug and replacement with an alternative medication. Compared to sudden withdrawal, measured outcomes were similar but gradual tapering was better tolerated. We conclude that for augmentation in RLS, gradual tapering of the augmentation-inducing dopaminergic drug is better tolerated than sudden withdrawal. The optimal approach to treating augmentation has not been established and may differ between patients. Further study with direct comparison of strategies and a larger patient population is needed to confirm our preliminary observations.
\end{abstract}

Keywords: restless legs syndrome, augmentation, treatment

\section{INTRODUCTION}

Restless legs syndrome (RLS) is a common condition that can disrupt sleep. While it can be treated with a variety of medications, dopaminergic drugs, including dopamine agonists and levodopa, appear to be the most reliable in alleviating symptoms. Unfortunately, however, long-term treatment with dopaminergics can lead to the problematic complication of augmentation [1,2]. Augmentation is a phenomenon in which the medication leads to a worsening of symptoms beyond the level of severity that was experienced when the medication was first given [1,2]. During augmentation, patients may experience an earlier onset of night time symptoms, more severe symptoms, the onset of day time symptoms and a declining response to the drug, requiring higher and higher doses. While most authorities recommend removing the offending drug [1,3,4], the best approach for accomplishing this or substituting for the drug has not been established. In a previous study, we found that sudden withdrawal of the dopaminergic medication with substitution of a medication from a different drug class (antiepileptics, benzodiazepines, analgesics) was generally poorly tolerated, resulting in a major worsening of symptoms and a long delay of many months before symptoms stabilized [5]. Because of that experience, we tested an alternative approach of very slowly tapering the dopaminergic drug while at the same time administering an alternative medication.

*Address correspondence to this author at The Atlantic Neuroscience Institute, Overlook Medical Center, 99 Beauvoir Ave. Summit, N.J. 07901; Tel: 908-522-2089; Fax: 908-522-6147;

E-mail: roger.kurlan@atlantichealth.org

\section{METHODS}

We conducted a retrospective chart review of RLS patients who presented to the Movement Disorders Program at the Atlantic Neuroscience Institute between 2010 and 2014 with dopaminergic drug-induced augmentation. We selected those patients who had the dose of their dopaminergic medication gradually tapered and stopped when possible and simultaneously treated with an alternative medication. We focused on the period of time from the recognition of augmentation to the stabilization of RLS symptoms. Findings were compared to our previous published experience with sudden dopaminergic drug withdrawal [5].

\section{RESULTS}

We identified 7 RLS patients who presented with dopaminergic drug-induced augmentation and were treated with this approach. There were five females and two males with a mean age of 71.7 (range 56-90) years. The mean time since diagnosis of RLS was 18 (range 3.5-45) years. At the time of appearance of augmentation, there were four patients taking regular or extended length ropinirole with a mean dose of 2.5 (range 1.0-3.5) $\mathrm{mg} / \mathrm{day}$, two patients taking regular or extended release pramipexole with a mean dose of 0.375 (range $0.25-0.5$ ) $\mathrm{mg} / \mathrm{day}$, and one patient taking controlled release carbidopa/levodopa at 50/200 mg/day. All of these medications had been taken at bedtime. The mean duration of dopaminergic drug therapy until the diagnosis of augmentation was 6.2 (range 0.5-20) years.

The treatment strategy for each patient was similar. Each began a gradual tapering schedule of their dopaminergic drug with the aim of trying to discontinue it over 3-6 months. 
All of the patients were given written tapering schedules to follow, decreasing the dose of their dopaminergic medication on a weekly or biweekly basis. At the same time, each patient was started on another RLS-suppressing drug. Gabapentin was prescribed as the substituted drug in 5 of the 7 cases and the dose was gradually increased as needed and as tolerated (final mean dose of $950 \mathrm{mg} /$ day; range 600 2100). In one case, clonazepam was the replacement drug (0.5 mg/day) and in another it was oxycodone/ acetaminophen (12.5/812.5 mg/day).

Of the 7 patients who began to wean off of the dopamine medication, 4 were able to stop the medication (mean time for discontinuation of 4.75 weeks; range $3-7$ ). Due to an inadequate response, 2 of the 4 patients required the addition of a second medication, including tramadol (50-100 mg/day) in 1 patient and clonazepam $(0.5 \mathrm{mg} /$ day $)$ in the other. The mean time to resolution of augmentation was 11.25 (range 223) weeks.

Three of the patients have not yet been able to complete their tapering of the dopaminergic drug and eliminate it due to recurrences of augmentation symptoms. At this time, mean duration of tapering is 32 (range 10-52) weeks. One patient required the addition of tramadol (50-125). The success of the gradual tapering for all of the patients varied greatly. For these patients, the most difficult stage has been stopping the drug from a small dosage. One patient was able to wean from $3.5 \mathrm{mg}$ of ropinirole to $1 \mathrm{mg}$ over 12 weeks without difficulty, but experienced a drastic increase in symptoms when decreasing from $1 \mathrm{mg}$ to $0.75 \mathrm{mg}$ and from $0.75 \mathrm{mg}$ to $0.5 \mathrm{mg}$, which took 16 weeks altogether. Another patient, who began at a dose of $1.0 \mathrm{mg}$ of ropinirole, has required 52 weeks to decrease to a dosage of $0.125 \mathrm{mg}$.

For the 7 patients viewed together, despite the long period of time sometimes needed for tapering, subjectively these patients have reported much less severe RLS symptoms than our prior group of patients treated with sudden withdrawal of the dopaminergic medication [5].

\section{DISCUSSION}

This study has limitations of being open-label, lacking RLS rating scale data and involving a small sample size. However, it is the first to report systematically gathered information on a group of RLS patients suffering from augmentation who were treated with a standard approach of gradually tapering the dopaminergic medication while replacing it with an alternative drug. We are able to compare the results of this approach to our report describing the poor response to sudden withdrawal of the offending agent previously published [5]. The sudden withdrawal and gradual tapering groups were similar clinically with mean age of 66 and 71 years, mean duration of RLS of 24 and 18 years, and mean duration of dopaminergic therapy prior to augmentation of 5.5 and 6 years, respectively. The outcomes of the two approaches turned out to be quite similar quantitatively with the mean time until augmentation resolved being 12.5 (range 1-43) weeks for those treated with sudden withdrawal and 11.2 (range 2-23) weeks for those treated with gradual titration. The latter group does not include the 3 patients who have not yet been able to stop the drug after a mean period of 32 (10-52) weeks. The primary difference between the two approaches was that subjectively, patients reported better tolerability with gradual titration, experiencing less severe worsening of RLS symptoms. It is possible that sudden withdrawal of a dopaminergic drug in RLS could precipitate a worsening of symptoms similar to what has been described in Parkinson's disease [6].

An apparent disadvantage of gradual tapering is that some of the patients have not yet been able to discontinue their dopaminergic drug over long time periods of up to a year. When examining the two patients who have had the most trouble discontinuing therapy, one had been taking ropinirole for 20 years before symptoms of augmentation appeared. This long duration of treatment may have contributed to the difficulty in withdrawing the medication. In another case, the patient had been treated with ropinirole for 5.5 years and had experienced a prior occurrence of augmentation and discontinued the medication. Two years later she restarted the drug leading to the recurrence of augmentation. Her difficulty coming off the drug may be due to the fact that this is her second attempt to deal with augmentation. In these cases, the simple addition of another drug type may have led to improvement in RLS symptoms.

For some patients, replacement of the dopaminergic drug with gabapentin was not straightforward. The majority required the use of a second medication in conjunction with gabapentin.

Our preliminary findings suggest that a slow taper off of the dopaminergic medication in patients experiencing augmentation is better tolerated than a sudden withdrawal of the drug. More formal research using direct, randomized comparisons and a larger sample size is needed to confirm this finding. It is clear that the treatment of augmentation is difficult and can take many months to accomplish. The optimal replacement drug in treating augmentation remains unsettled. The response to dopaminergic drug withdrawal or tapering appears to differ substantially across patients. The optimal approach for treating augmentation remains to be established and may differ from person to person.

\section{ACKNOWLEDGEMENTS}

Declared none.

\section{AUTHORS' ROLES}

Mr. Rosenstein- Execution of research, writing first draft.

Dr. Rabin- conception, organization of research, review of manuscript.

Dr. Kurlan-Conception, organization of research, review and critique of manuscript.

\section{FINANCIAL DISCLOSURES}

Related to research in this article: None. No conflicts.

Previous 12 months:

Rosenstein- None 
Rabin- Speakers bureau for Teva, Ipsen. Research support from Ipsen.

Kurlan- Speakers bureau for Teva. Research support from Psyadon, Otsuka, Astra-Zeneca.

\section{REFERENCES}

Kurlan R, Richard IH, Deeley C. Medication tolerance and augmentation in restless legs syndrome: the need for drug class rotation. J Gen Intern Med. 2006;21:C1-4.

[2] Garcia-Borreguero D, Hogl B, Ferini-Strambi L, Winkelman J, Hill-Zabala C, Asgharian A, et al. Systematic evaluation of augmentation during treatment with ropinirole in restless legs syndrome (Willis-Ekbom disease): results from a prospective, multicenter study over 66 weeks. Movement Dis 2012; 27: 277-83.

[3] Williams A-M, Garcia-Borreguero D. Management of restless legs syndrome augmentation. Curr Treat Options Neurol 2009; 11:32732 .

[4] Oertel WH, Trenkwalder C, Zucconi M, Benes H, GarciaBorreguero D, Bassetti C, et al. State of the art in restless legs syndrome therapy: Practice recommendations for treating restless legs syndrome. Movement Disorders 2007; 22(S18):S466-75.

[5] Kurlan R, Rabin M. Augmentation in restless legs syndrome: Poor response to sudden withdrawal of dopaminergic therapy. J Parkinsonism Restless Legs Synd 2013; 3:49-52.

[6] Rabinak CA, Nirenberg MJ. Dopamine agonist withdrawal syndrome in Parkinson disease. Arch Neurol 2010;67: 58-63.

Received: January 10, 2014

Revised: March 24, 2014

Accepted: March 25, 2014

(C) Rosenstein et al.; Licensee Bentham Open.

This is an open access article licensed under the terms of the Creative Commons Attribution Non-Commercial License (http://creativecommons.org/licenses/ by-nc/3.0/) 